Cerebrovasc Dis 2007;24:152-154

DOI: 10.1159/000103622

\section{Emergency Surgical Revascularization in a Patient with Acute Symptomatic Common Carotid Artery Occlusion}

OlafCrome a , Pawel Kermer a, Jan-Hendrik Buhk ${ }^{b}$, Friedrich Schoendube ${ }^{c}$, Andreas Kastrup ${ }^{a}$

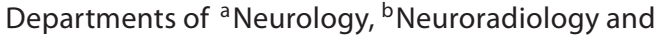
'Thoracic Cardiovascular Surgery, University of Göttingen, Göttingen, Germany

\section{Introduction}

Isolated common carotid artery (CCA) occlusion with patent internal and external carotid arteries (ICA/ECA) is a rare event, which is often associated with recurrent ischemia due to arteryartery thromboembolism [1]. However, in a subset of these patients, large brain regions may additionally be compromised hemodynamically leading to a fluctuating and ultimately progressive clinical course. While some authors have reported successful surgical revascularization in selected subacute or chronic patients with clinical fluctuations secondary to CCA occlusion with hemodynamic instability in the past $[1,2]$, the value of this procedure as an acute therapeutic option is unknown. Here, we report our experience on a patient treated with subclavian artery to distal CCA bypass during the acute phase of a symptomatic CCA occlusion.

\section{Case Report}

A 55-year-old male patient with a history of arterial hypertension and hyperlipidemia attended his local hospital with minor difficulties in word finding and a moderate right hemiparesis both of sudden onset (NIHSS 2, mRS 2). Magnetic resonance imaging (MRI) revealed a single, small infarction in the left MCA territory and an occlusion of the left CCA. Due to fluctuating symptoms the patient was transferred to our department. While his initial clinical assessment was unchanged at admission, MRI now demonstrated multiple thromboembolic infarctions in the left MCA territory and confirmed the presence of an isolated leftsided CCA occlusion with patent internal and external carotid arteries (fig. 1). Additionally, a severe perfusion deficit in the entire left MCA territory and to a lesser extent in both PCA territories was shown by CT perfusion imaging. According to an extracranial and transcranial color-coded duplex sonography study, this perfusion deficit was due to a combination of (a) left CCA occlusion, (b) hypoplasia in the left A1 segment and (c) insufficient collateralization from posterior circulation caused by a combination of a high-grade stenosis at the origin of the left vertebral artery (VA) and hypoplasia of the opposite VA resulting in decreased basilar artery flow. Extracranially, ultrasound examination displayed signs of a diffuse atherosclerotic disease and revealed fresh
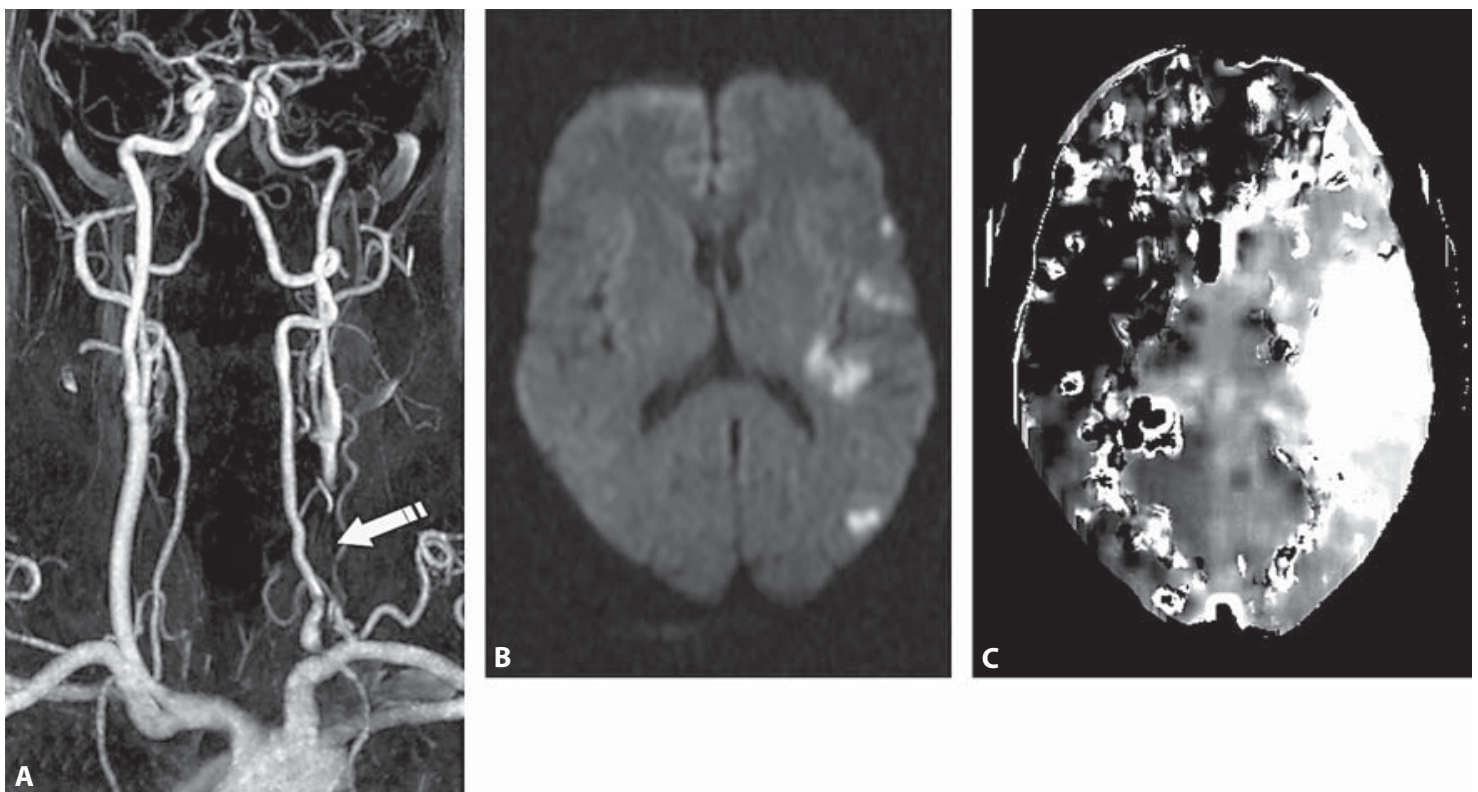

Fig. 1. Preoperative diagnostic workup with MRI and CT perfusion (CTP) imaging. A Contrast-enhanced MR angiography (CE-MRA) confirms the presence of an isolated left-sided CCA occlusion (arrow) with patent internal and external carotid arteries. B Diffusion-weighted MRI (DWI, b =1,000) demonstrates multiple thromboembolic infarctions in the left MCA territory. C CTP (mode: time to peak/TTP) reveals a severe perfusion deficit in the entire left MCA territory and to a lesser extent in both PCA territories (white pixels refer to longer TTP). 
Fig. 2. Postoperative CT imaging. A CT angiography demonstrates revascularization performed by a subclavian artery to distal CCA bypass (arrows); the occluded proximal CCA is ligated and not visible in CTA. B Perfusion (TTP) of the left MCA territory was restored except for a small area of infarction (white pixels refer to longer TTP).
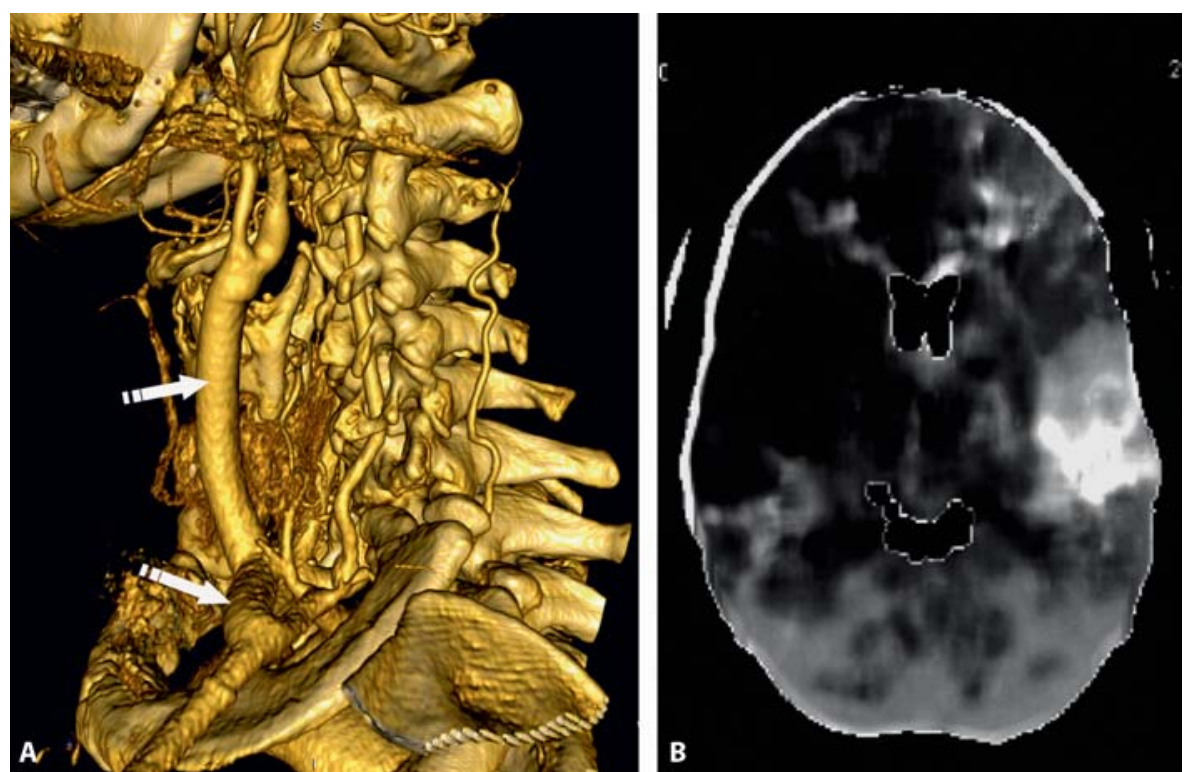

thrombotic material in the occluded left CCA as the source of the occlusion and recurrent thromboembolic events. In the patent ECA a retrograde flow was present giving rise to a minimal residual anterograde ICA flow. Due to the presence of fresh thrombotic material in the CCA, anticoagulation by continuous heparin application was initiated. Further diagnostic work-up excluded the presence of a dissection within the aortic arch as the cause of CCA occlusion.

Shortly after admittance the patient repetitively experienced dramatic worsening of his neurological symptoms including global aphasia and right hemiplegia depending on his blood pressure. Since these recurrent events could only be prevented by continuous blood pressure elevation with catecholamine exposure above a threshold value of approximately $180 / 100 \mathrm{~mm} \mathrm{Hg}$, urgent cerebral revascularization was performed 9 days after the initial event by a subclavian artery to distal CCA bypass (fig. 2). Intraoperative continuous transcranial Doppler monitoring of the MCA flow confirmed the presence of an insufficient collateral circulation. In fact, MCA flow on the left side was completely abolished during clipping of the distal ICA. Due to the completely abolished flow in the MCA on the left side during clipping, the initial intention to perform a local endarterectomy and thrombectomy of the CCA was abandoned. Instead, a bypass from the subclavian artery (end-to-side anastomosis) to the CCA (end-toend anastomosis) was implanted using a knitted double velour vascular graft with a diameter of $8 \mathrm{~mm}$ and length of $15 \mathrm{~cm}(\mathrm{He}-$ mashield Gold, Boston Scientific, N.J., USA). The total clamping time of the internal carotid artery during this procedure was 14 min. Perfusion of the left hemisphere was restored and the occluded proximal CCA was ligated in order to prevent further thromboembolic events (fig. 2). Duplex sonography revealed a low but sufficient flow in the bypass, and a sufficient flow in the left ICA, ECA as well as MCA. The patient was discharged 14 days later with an NIHSS of 0 and a Modified Rankin Scale of 1.

\section{Discussion}

We have presented a 55-year-old male patient with acute symptomatic CCA occlusion, in whom urgent surgical revascularization was performed to improve insufficient hemispherical blood flow and thus to prevent progressive stroke. While previous reports have demonstrated the feasibility of subclavian to common or internal carotid artery bypass surgery in selected patients with subacute or chronic CCA occlusion $[1,2]$, our rare case supports the notion that this procedure is also feasible and effective in acute stroke patients with repeated post-occlusive thromboembolic events as well as with severe hemodynamic insufficiency.

In general, isolated CCA occlusion with ICA and ECA patency is most commonly due to a thrombosis of a local atherosclerotic plaque. After excluding other rare causes of isolated CCA occlusion such as aortic dissection on the one hand and finding signs of a generalized atherosclerosis on the other, this was also the most probable cause in our patient. Although the incidence of an isolated CCA occlusion in acute stroke patients is low, it is associated with an extremely high risk of recurrent ischemic events due to both repeated post-occlusive thromboembolic events and insufficient cerebral hemodynamics [1], stressing the importance of rapid identification of this potentially devastating disorder. Comparable with other previous case reports $[3,4]$, extracranial and transcranial color-coded duplex sonography was effective in visualizing the patent distal vessels in the presence of a CCA occlusion. In our patient, a minimal residual anterograde flow in the ICA was preserved by a retrograde flow from the ECA into this vessel, whereas in the face of a reduced basilar artery flow due to a high-grade stenosis at the origin of the left VA and hypoplasia of the opposite VA the intracranial collateral flow was insufficient to maintain an adequate blood supply to the left hemisphere, as evidenced by additional CT perfusion imaging. These findings serve as a good explanation for the high susceptibility to blood pressure fluctuations seen in our patient. In fact, a dramatic neu- 
rological deterioration was only prevented by pharmacologically induced elevation of blood pressure, supporting the need of an urgent revascularization to prevent additional permanent damage. At least partially, cerebral perfusion to the left hemisphere could also have been improved by stenting the high-grade stenosis at the origin of the left VA. While we did not find any reports on emergency bypass surgery of an occluded CCA, we encountered several case reports and small studies dealing with both successful emergency thrombendarterectomy or angioplasty and stenting of an occluded ICA $[5,6]$. In contrast to an endovascular approach, in our case the surgical approach offered the opportunity to ligate the CCA to prevent further embolic events, which often arise from the stump of an occlusion [7]. Despite the lack of a generalized efficacy of bypass surgery for occlusive carotid disease [8], emergency surgical recanalization of an acute atherothrombotic CCA occlusion is technically feasible and holds promise to avoid major stroke in selected cases with insufficient cerebrovascular collateralization or repeated post-occlusive thromboembolic events.

\section{References}

1 Belkin M, Mackey WC, Pessin MS, Caplan LR, O'Donnell TF: Common carotid artery occlusion with patent internal and external carotid arteries: diagnosis and surgical management. J Vasc Surg 1993;17: 1019-1027.

2 Archie JP Jr: Axillary-to-carotid artery bypass grafting for symptomatic severe common carotid artery occlusive disease. J Vasc Surg 1999; 30:1106-1112.

3 Cull DL, Hansen JC, Taylor SM, Langan EM III, Snyder BA, Coffey CB: Internal carotid artery patency following common carotid artery occlusion: management of the asymptomatic patient. Ann Vasc Surg 1999;13:73-76.

4 Dermitzakis I, Minardos I, Kampanarou M, Mitakou D: Color duplex sonography of occlusion of the common carotid artery with reversed flow in the extracranial internal carotid artery. J Clin Ultrasound 2002; 30:388-391.

5 Jovin TG, Gupta R, Uchino K, Jungreis CA, Wechsler LR, Hammer MD, Tayal A, Horowitz MB: Emergent stenting of extracranial internal carotid artery occlusion in acute stroke has a high revascularization rate. Stroke 2005;36:2426-2430.

6 Meyer FB, Sundt TM Jr, Piepgras DG, Sandok BA, Forbes G: Emergency carotid endarterectomy for patients with acute carotid occlusion and profound neurological deficits. Ann Surg 1986;203:82-89.

7 Barnett HJ, Peerless SJ, Kaufmann JC: 'Stump' on internal carotid artery - a source for further cerebral embolic ischemia. Stroke 1978;9: 448-456.

8 EC/IC Bypass Study Group: Failure of extracranial-intracranial arterial bypass to reduce the risk of ischemic stroke. Results of an international randomized trial. N Engl J Med 1985;313:1191-1200.

Andreas Kastrup, MD

Department of Neurology, University of Göttingen

Robert-Koch-Strasse 40

DE-37075 Göttingen (Germany)

Tel. +49551396 603, Fax +49551398405

E-Mail andreas.kastrup@medizin.uni-goettingen.de
Cerebrovasc Dis 2007;24:154-155

DOI: $10.1159 / 000103623$

\section{Cardiac Hemangioma of the Right Atrium: A Possible Cause of Cerebellar Stroke}

\begin{abstract}
Stefano Pasquino a , Clotilde Balucani ${ }^{\mathrm{b}}$, Isidoro di Bella ${ }^{\mathrm{a}}$, Andrea Alberti ${ }^{\mathrm{b}}$, Uberto da $\mathrm{Col}^{\mathrm{a}}$, Valeria Caso ${ }^{\mathrm{b}}$, Enrico Ramoni ${ }^{\mathrm{a}}$, Maurizio Paciaroni ${ }^{\mathrm{b}}$, Giancarlo Agnelli ${ }^{\mathrm{b}}$, Temistocle Ragni ${ }^{\mathrm{a}}$

a Department of Cardiac Surgery, Azienda Ospedaliera di Perugia, and ${ }^{b}$ Department of Internal Medicine, Stroke Unit, University of Perugia, Perugia, Italy
\end{abstract}

Case

We report on a possible paradoxical embolization via interatrial defect (IAD) from a cavernous hemangioma of the right atrium leading to a cerebellar stroke. This is an unusual clinical presentation with a location that is uncommon for this rare type of heart tumor [1].

A 57-year-old man was admitted to our hospital with an acute transitory syndrome: sudden bilateral tinnitus and vertigo followed by onset of coma with a Glasgow Coma Scale score of 3 lasting around $10 \mathrm{~min}$. After this, the patient was alert and the neurological examination normal. Continuous ECG monitoring showed stable sinus rhythm, while diffusion-weighted brain MRI (DWI-MRI) scans were negative. During hospitalization the patient developed nausea and vomiting, saccadic eye movements, an unidirectional right beating nystagmus of the primary and bilateral gaze positions and left mild hemiataxia. DWI-MRI revealed a small ischemic infarction of the left superior cerebellar artery territory. Thrombophilic screening, neoplastic markers, autoimmune assays and cerebral angiography were negative. An acute coronary syndrome, without coronary stenoses, had occurred 4 years earlier, so aspirin was started.

Transesophageal echocardiography revealed a sessile neoformation inside the right atrium and on the posterior part of the interatrial septum, which caused a partial obstruction of the inferior vena cava (IVC) orifice. The remaining anterosuperior interatrial septum appeared to be aneurysmatic with an IAD inside the area of the fossa ovalis. During Valsalva maneuver right to left shunt was evidenced. No other cardioembolic lesions (i.e. aortic plaques, left atrial appendage thrombi) were found. Embolic cardiac right atrial myxoma was suspected. Coronary angiography documented neither significant atherosclerotic lesions nor vascular distortions. Eighteen days after stroke onset, the tumor was surgically removed. An oval, red-brown colored, elastic, uniformly endothelized, sessile cardiac neoplasm (4 cm in diameter) was removed. There was no evidence of ulceration or clot adhesion on the surface (fig. 1).

The cardiac tumor had a wide base attachment at the level of the inferior part of the interatrial septum, near the IVC orifice, which partly obstructed the IVC orifice. The wide implant surface and elastic consistence did not lead to occlusion of the IVC or tricuspid valve. The mass was resected and IAD closed by direct suture; histological examination revealed it to be a cardiac cavernous hemangioma (fig. 2). The patient was discharged on the 7 th postoperative day. At the 6-month follow-up no vascular 